\title{
Visusverlust durch Adalimumab-Therapie bei Plaque-Psoriasis
}

\author{
Norman A. Saffra Konstantin Astafurov \\ Maimonides Medical Center, Brooklyn, NY, USA
}

Schlüsselwörter

Adalimumab · Psoriasis · Optikusneuritis · Demyelinisierung

Visusverlust

\section{Zusammenfassung}

Bei einem 61-jährigen Patienten kaukasischer Abstammung mit schwerer Plaque-Psoriasis ohne Gelenkbeteiligung wurde eine Behandlung mit Adalimumab eingeleitet. Kurz darauf stellte er sich aufgrund akuter Sehverschlechterung im rechten Auge in der Notaufnahme vor. Eine umfassende systemische Untersuchung wurde durchgeführt, einschließlich Magnetresonanztomographie (MRT)Aufnahmen des Gehirns und der Orbitae mit und ohne Gadoliniumverstärkung. Der MRT-Befund war vereinbar mit ZNS-Demyeli- nisierung und Retrobulbärneuritis. Adalimumab wurde sofort abgesetzt und keine weitere systemische Therapie eingeleitet. 6 Wochen später war das Sehvermögen des Patienten vollständig wiederhergestellt. Weitere Psoriasis- oder neurologische Behandlungsmaßnahmen erfolgten nicht. Der Patient ist seit 14 Monaten stabil.

(c) 2017 S. Karger GmbH, Freiburg

\section{Fallbericht}

Der 61-jährige Patient kaukasischer Abstammung leidet an Plaque-Psoriasis ohne Gelenkbeteiligung. In der Notaufnahme klagte er primär über zunehmende Sehverschlechterung seit 5 Tagen und über schmerzende Augenbewegungen. Seine ophthalmologische Vorgeschichte, die sonstige Krankengeschichte sowie die Besprechung der Symptomatik gaben keinen weiteren Aufschluss. Signifikant in seiner dermatologischen Krankengeschichte war das Versagen einer Psoriasistherapie mit Etanercept. Vor seiner Vorstellung wurde der Patient seit 8 Wochen mit Adalimumab behandelt, mit klinischer Verbesserung der Psoriasis.

Eine sofortige augenärztliche Untersuchung ergab als signifikante Befunde eine Verschlechterung der Sehschärfe von 20/50 im rechten Auge mit afferentem Pupillendefekt und vermindertem Farbsehen auf der rechten Seite; die Funduskopie war unauffällig. Eine formale Gesichtsfeldmessung ergab einen bogenförmigen
Gesichtsfelddefekt im rechten Auge und keine Ausfälle im linken Auge (Abb. 1). Umfassende Laboruntersuchungen einschließlich Fluoreszenz-Treponema-Antikörper-Absorption-Test (FTA-Abs) und Rapid-Plasma-Reagin-Test (RPR) ergaben Werte im Normbereich. Auf MRT-Aufnahmen des Gehirns und der Orbitae mit und ohne Gadoliniumverstärkung zeigten sich keine orbitalen Anomalien, jedoch in beiden Hemisphären multiple unspezifische Hyperintensitäten in der weißen Hirnsubstanz in FLAIRund T2-gewichteten Sequenzen ohne Masseneffekt oder Ödembildung. Diese Ergebnisse waren vereinbar mit ZNS-Demyelinisierung und Retrobulbärneuritis. Adalimumab wurde sofort abgesetzt. Eine formale neurologische Konsultation ergab die Empfehlung zur Einleitung einer systemischen Steroidtherapie, die der Patient jedoch ablehnte.

In der augenärztlichen Kontrolluntersuchung 6 Wochen später waren Sehschärfe und Gesichtsfeld vollständig wiederhergestellt (Abb. 2) und blieben in den nächsten 14 Monaten stabil. Die Psoriasis wurde nicht wieder systemisch behandelt.

\section{KARGER \\ Fax +497614520714 \\ information@karger.com}

www.karger.com

\section{(c) 2017 S. Karger GmbH, Freiburg}

Accessible online at:

www.karger.com/kkd
Norman A. Saffra, MD

Maimonides Medical Center

902 49th Street, Brooklyn, NY 11219, USA

eyesitemd@gmail.com 
Abb. 1. Die Gesichtsfeldmessung (SITA 24-2) bei der Erstvorstellung ergab einen superioren bogenförmigen Defekt (Pfeil) im rechten Auge.

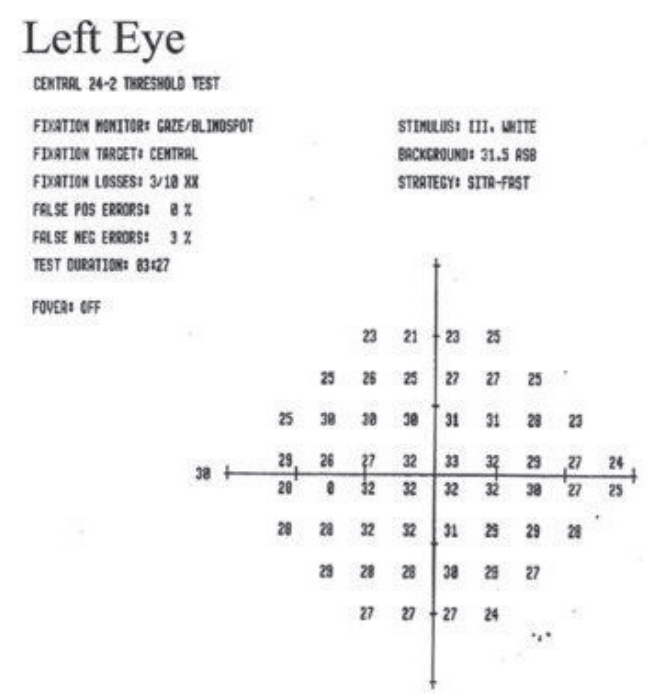

\section{Right Eye}

central 24-2 natswalo trst

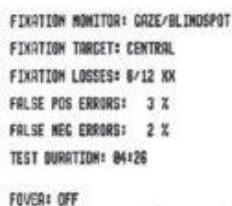

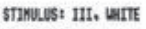
Backerouvo: 31.5 288 STRRTEGT: STRA-FAST

\begin{tabular}{|c|c|}
\hline 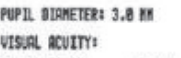 & $\begin{array}{l}\text { DATE: 11-23-2214 } \\
\text { TIKE: SMB PH }\end{array}$ \\
\hline $2 x: 40.75$ as & REE: 61 \\
\hline
\end{tabular}

Qx: 4.75 os $x \times x$

QCE: 61
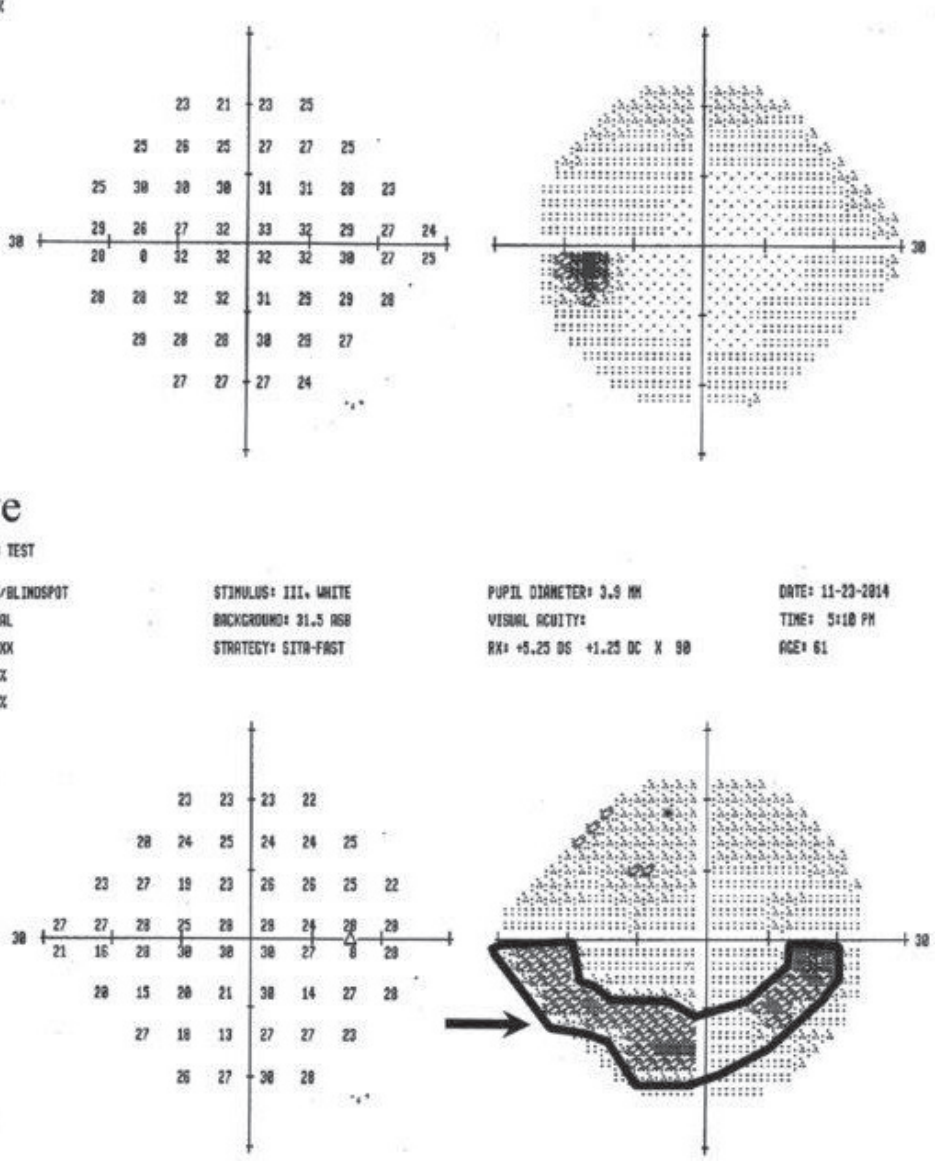

visiel scuitr: RX1 +5.25 os +1.25 oc $\times$ 98

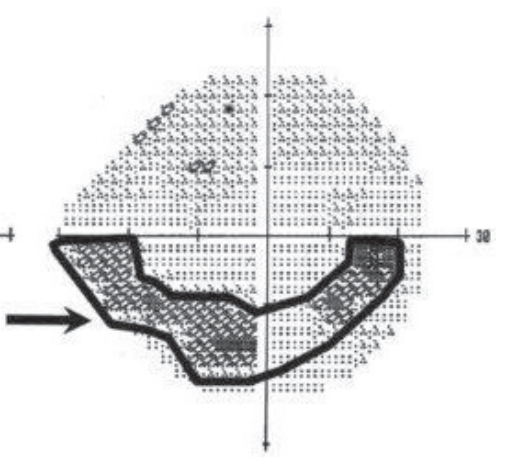

\section{Diskussion}

Adalimumab ist ein rekombinanter humaner monoklonaler Antikörper, der den Tumornekrosefaktor-alpha (TNF- $\alpha$ ) neutralisiert. Er ist von der US-Zulassungsbehörde Food and Drug Administration (FDA) zur Behandlung der mittelschweren bis schweren Psoriasis sowie einer Reihe anderer Autoimmun- und rheumatologischer Erkrankungen zugelassen. Die Anwendung von TNF- $\alpha-H e m m e r n$ einschließlich Adalimumab ist mit verschiedenen demyelinisierenden Erkrankungen sowohl des zentralen als auch des peripheren Nervensystems in Verbindung gebracht worden. Es gibt Fallberichte über Retrobulbärneuritis, Multiple Sklerose und Querschnittsmyelitis sowie das GuillainBarré-Syndrom im Zusammenhang mit dem Einsatz von TNFa-Hemmern bei verschiedenen rheumatologischen und entzündlichen gastrointestinalen Erkrankungen [1-3]. Wir berichten hier erstmals über einen Fall von Retrobulbärneuritis und ZNS-Demyelinisierung bei einem Patienten, der wegen Plaque-
Psoriasis ohne Gelenkbeteiligung mit Adalimumab behandelt wurde.

Zielgerichtete biologische Therapien, die bei T-Helferzellen und Immunzytokinen ansetzen, werden dank ihrer klinischen Wirksamkeit und relativen Sicherheit in den letzten Jahren vermehrt zur Behandlung von Psoriasis und Psoriasis-Arthritis eingesetzt [4]. TNF- $\alpha$-Inhibitoren wie Adalimumab sind die meistverwendeten Biologika bei diesen Indikationen. Sie sind nicht nur mit einem erhöhten Infektionsrisiko assoziiert, sondern auch mit einem potenziellen Risiko für das Auftreten einer demyelinisierenden Erkrankung des zentralen oder peripheren Nervensystems bzw. mit dem Risiko einer Verschlechterung, wenn eine solche Erkrankung bereits besteht [1-3]. In einer aktuellen Studie wurden sämtliche bisher vorliegenden Berichte über demyelinisierende Erkrankungen im Zusammenhang mit einer Anti-TNF- $\alpha$ Therapie bei Psoriasis und Psoriasis-Arthritis analysiert. Die Autoren gelangten zu dem Schluss, dass diese Komplikationen recht selten sind, da bisher erst über 21 weitere Fälle während und nach 
Abb. 2. Die Gesichtsfeldmessung (SITA 24-2) bei der Kontrolluntersuchung nach 6 Wochen zeigte die beidseitige vollständige Wiederherstellung des Gesichtsfelds.

\section{Left Eye}

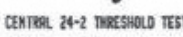

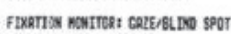

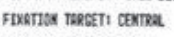

FXxition Lessst e 13

False pas ERoces: of

FALSE KEG EROQS: $\mathrm{x}$

TEST xearion: 20:58

FOEER: OFF
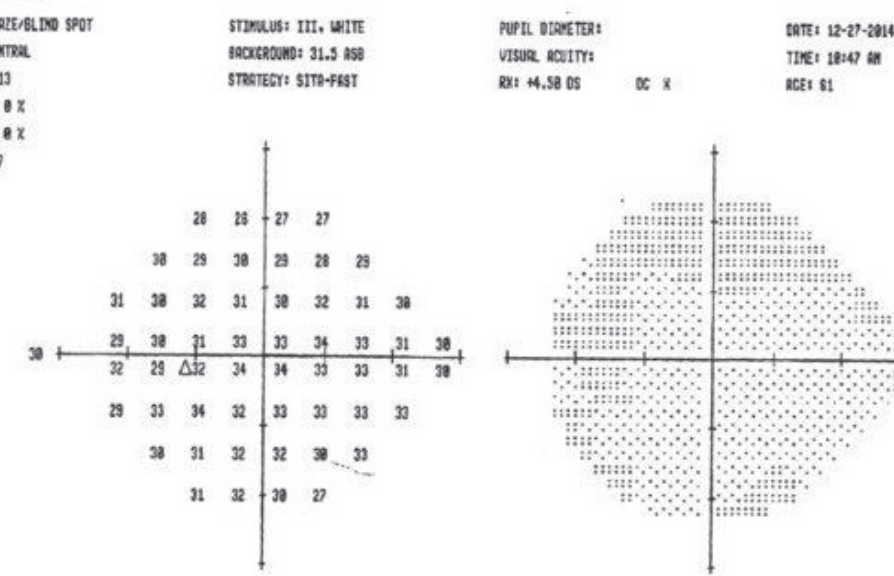

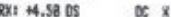

RCEt 81

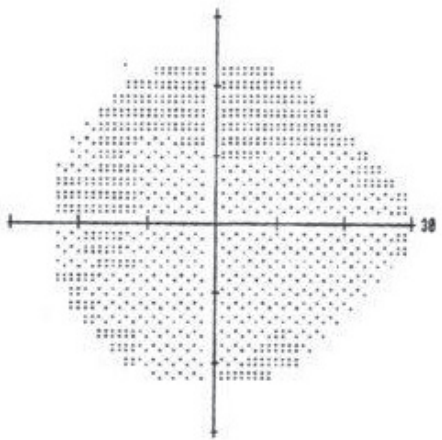

\section{Right Eye}

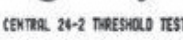

FDXatrox notTort corz/ate SPOT

FDatras Trocels: cenrra.

FDartara tosses: $2 / 11$

strmus: III. Hitt:

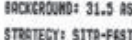

FR.SE POS EXovs: $7 \%$ FRLSE NES BRQNeS:

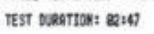
FOVER: off

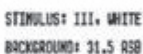
BaCkerouve: 31.5 RSB
STRRTEEY: SITR-FaST 2

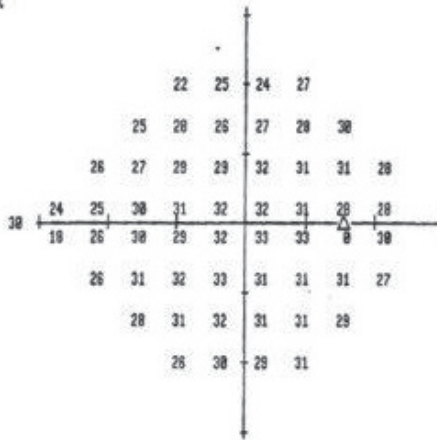

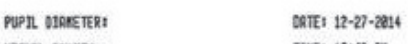
VISPL ACUIT: TINE! 18010 on $2 \times 1+6.75$ of -2.25 oC $\times 24 \quad$ aCE1 61

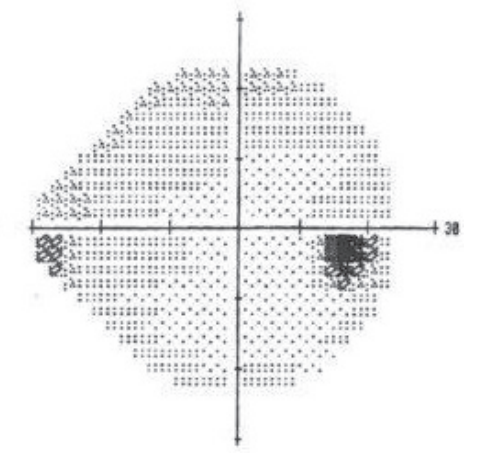

klinischen Studien berichtet worden ist [5]. Wir beschreiben in unserem Fallbericht erstmals die Assoziation einer AdalimumabTherapie mit Retrobulbärneuritis und ZNS-Demyelinisierung bei einem Patienten mit Plaque-Psoriasis ohne Psoriasis-Arthritis. Eine kontinuierliche Überwachung auf mögliche neurologische Nebenwirkungen ist bei Psoriasis-Patienten unter Adalimumab-Therapie möglicherweise angezeigt.

\section{Literatur}

1 Bensouda-Grimaldi L, Mullemann D, Valat JF, Autret-Leca E: Adalimumab-associated multiple sclerosis. J Rheumatol 2007;34:239-240.

2 Shin IS, Baer AN, Kwon HJ, Papadopoulos EJ, Siegel JN: Guillain-Barre and Miller Fisher syndromes occurring with tumor necrosis factor alpha antagonist therapy. Arthritis Rheum 2006;54:1429-1434.
3 Chung JH, Van Stavern GP, Frohman LP, Turbin RE: Adalimumab-associated optic neuritis. J Neurol Sci 2006;244:133-136.

4 Weger W: Current status and new developments in the treatment of psoriasis and psoriatic arthritis with biological agents. Br J Pharmacol 2010;160:810-820.
5 Zhu TH, Nakamura M, Abrouk M, Farahnik B, Koo J, Bhutani T: Demyelinating disorders secondary to TNF-inhibitor therapy for the treatment of psoriasis: a review. J Dermatolog Treat 2016;27:406-413. 